\title{
Estudio Comparativo de Clinkers Producidos con Diferentes \\ Reemplazos de Combustibles Residuales
}

\author{
Mónica Adriana Trezza ${ }^{\mathrm{a} *}$, Alberto Néstor Scian ${ }^{\mathrm{b}}$ \\ ${ }^{a}$ Facultad de Ingenieria - UNCPBA. \\ Av. del Valle 5737 (B7400JWI) Olavarría, Argentina \\ ${ }^{\mathrm{b}}$ Centro de Tecnología de Recursos Minerales y Cerámica, CONICET, UNLP. \\ $C^{\text {no }}$ Centenario y 506 (1897) - Gonnet, Argentina
}

Received: November 11, 2001; Revised: March 05, 2003

\begin{abstract}
In the last decades the cement industry has used, as a partial substitute for traditional fuel, different industrial wastes in order to profit their residuals energy. In the present work the physicochemical characteristics of clinker, obtained using trade marked alternative fuel for clinker kiln, were evaluated. X-ray diffraction (XRD), Blaine specific surface, pyrometric cone equivalent (PCE), differential thermal analysis-thermal gravimetry simultaneous (DTA-TG), differential calorimetry, porosimetry and mechanical strength were used as the main evaluation techniques of the characteristic of clinker and their behaviors in the early hydration. The additions of the wastes, within the percentages and under the conditions of this test, does no significantly alter the clinker properties but generates a greater crystallinity of the principal clinker phases.
\end{abstract}

Keywords: co-procesamiento, residuos combustibles, clinker, combustibles alternativos.

\section{Introducción}

La utilización de residuos combustibles en hornos de cemento es una práctica válida en todo el mundo y se perfila como una alternativa muy interesante en nuestro país. Especialmente considerando que las industrias Argentinas generan gran cantidad de residuos que en muchos casos están siendo almacenados sin control ni recaudo alguno o se les da un destino incierto.

A partir de los años '70 las empresas ligadas a la gestión de residuos y las industrias cementeras inician una etapa de co-procesamiento de residuos peligrosos a fin de valorizar los mismos al utilizar el poder calorífico residual de estos desechos como combustible secundario en sus hornos de clinker. El blending ó combustible mezcla obtenido posee especificaciones determinadas que aseguran un poder calorífico constante y bajo contenido de cloruros. El mismo puede alimentar al sistema central de calentamiento conjuntamente con el combustible principal a través de un diseño especial del quemador.

Las altas temperaturas en el quemador, el tiempo de re- sidencia de los gases en el horno y el contenido de oxígeno aseguran la combustión completa de los compuestos orgánicos aún los más estables. En cuanto a los compuestos inorgánicos y metales pesados -que eventualmente pueden contener los residuos- se combinarían con los silicatos, o quedarían atrapados en la fase vítrea que se forma durante la clinkerización, convirtiendo los compuestos tóxicos en inofensivos o menos nocivos ${ }^{1}$. Se constituye así la matriz de cemento portland en un lugar adecuado para la solidificación/estabilización (S/E) de residuos peligrosos evitando su enterramiento o quema con las consecuencias conocidas.

Diferentes autores ${ }^{2-11}$ han estudiado este punto, encontrando que la matriz de cemento Portland normal ó con adiciones es adecuada para la $\mathrm{S} / \mathrm{E}$ de metales como $\mathrm{Zn}, \mathrm{Cu}$, $\mathrm{Pb}, \mathrm{Cd}$ entre otros. Reportan además, la formación de fases intermedias en el sistema $\mathrm{CaO}-\mathrm{SiO}_{2}-\mathrm{Al}_{2} \mathrm{O}_{3}$-óxido metálico, que son estabilizadas durante la clinkerización y/o hidratación del cemento portland ${ }^{13-15}$, encontrando que en todos los casos el comportamiento depende de las condiciones del sistema y de las concentraciones de los contami-

*e-mail: mtrezza@ fio.unicen.edu.ar 
nantes. Sin embargo, no estudian el efecto conjunto de diferentes metales en concentraciones variables, tal como se incorporan a través de los combustibles residuales.

Se presentan en este trabajo los estudios realizados sobre diferentes clinkers elaborados en condiciones de laboratorio, con distintos niveles de adición de residuos tal como se incorporarían por el reemplazo parcial del combustible. Los porcentajes de reemplazo se mantuvieron dentro de los límites aceptados en las plantas industriales. Los sistemas analizados en este estudio surgen de la clinkerización de polvo crudo en presencia una mezcla combustible de marca registrada usada actualmente como combustible alternativo en la industria cementera. Los resultados se compararán con los correspondientes a un clinker obtenido sin este tipo de incorporación (M0).

Se estudiaron las características del clinker y su comportamiento durante la hidratación temprana por técnicas de difracción de rayos X (DRX), medición de superficie específica Blaine, temperatura de cono pirométrico equivalente (TCPE), análisis térmico diferencial y termogravimétrico simultáneo (ATD/TG), calorimetría diferencial, porosimetría por intrusión de mercurio y resistencia mecánica a la compresión.

\section{Experimental}

\subsection{Materiales}

El material crudo utilizado en este trabajo corresponde a una mezcla industrial provista por una cementera local. Su análisis químico, realizado por Fluorescencia de rayos $\mathrm{X}$ se presenta en la Tabla 1.

El material fue caracterizado por DRX. Las fases cristalinas mayoritarias observadas fueron $\mathrm{CaCO}_{3}$ (calcita) y $\mathrm{SiO}_{2}$ (cuarzo). Entre los constituyentes cristalinos minoritarios se detectó muscovita $\left[\mathrm{KAl}_{2} \mathrm{Si}_{3} \mathrm{AlO}_{10}(\mathrm{OH})_{2}\right]$.

\subsection{Preparación de las muestras}

En nuestro país los combustibles alternativos están reemplazando aproximadamente el $20 \%$ del combustible tradicional. Sabiendo que por cada $100 \mathrm{~kg}$ de clinker se necesitan aproximadamente $10 \mathrm{~kg}$ de combustible equivalente en carbono, se podrá utilizar hasta $2 \mathrm{~kg}$ de estos combustibles alternativos. Los porcentajes de sustitución varían según el país ${ }^{16}$.

De acuerdo a estos niveles de reemplazos se prepararon para este estudio diferentes mezclas de polvo crudo y se incorporaron directamente las cenizas dejadas por el combustible después de su combustión total. El análisis químico de los principales componentes de las cenizas expresado como óxidos se muestra en la Tabla 2 , se incluye además los contenidos de los metales mayoritariamente encontrados en las mismas.

Para este caso se prepararon muestras con tres niveles
Tabla1. Composición centesimal del polvo crudo, como óxidos

\begin{tabular}{llll}
\hline $\mathrm{CaO}$ & 43,52 & $\mathrm{~K}_{2} \mathrm{O}$ & 0,69 \\
\hline $\mathrm{SiO}_{2}$ & 14,28 & $\mathrm{Na}_{2} \mathrm{O}$ & 0,03 \\
\hline $\mathrm{Al}_{2} \mathrm{O}_{3}$ & 3,12 & $\mathrm{SO}_{3}$ & 0,49 \\
\hline $\mathrm{Fe}_{2} \mathrm{O}_{3}$ & 2,37 & P.P.C. & 34,9 \\
\hline
\end{tabular}

Tabla 2. Composición centesimal de las cenizas de la mezcla combustible.

\begin{tabular}{llll}
\hline $\mathrm{CaO}$ & $7,22 \%$ & $\mathrm{Co}$ & $1100 \mathrm{ppm}$ \\
\hline $\mathrm{SiO}_{2}$ & $49,13 \%$ & $\mathrm{Cr}$ & $905 \mathrm{ppm}$ \\
\hline $\mathrm{Al}_{2} \mathrm{O}_{3}$ & $15,09 \%$ & $\mathrm{Cu}$ & $6850 \mathrm{ppm}$ \\
\hline $\mathrm{Fe}_{2} \mathrm{O}_{3}$ & $12,44 \%$ & $\mathrm{~Pb}$ & $36500 \mathrm{ppm}$ \\
\hline $\mathrm{MgO}$ & $1,48 \%$ & $\mathrm{Zn}$ & $25700 \mathrm{ppm}$ \\
\hline $\mathrm{Na}_{2} \mathrm{O}$ & $2,48 \%$ & $\mathrm{Mo}$ & $123 \mathrm{ppm}$ \\
\hline $\mathrm{K}_{2} \mathrm{O}$ & $2,77 \%$ & $\mathrm{Ni}$ & $266 \mathrm{ppm}$ \\
\hline $\mathrm{TiO}_{2}$ & $1,93 \%$ & $\mathrm{Sb}$ & $152 \mathrm{ppm}$ \\
\hline $\mathrm{MnO}_{2}$ & $0,12 \%$ & $\mathrm{Sr}$ & $380 \mathrm{ppm}$ \\
\hline $\mathrm{P}_{2} \mathrm{O}_{5}$ & $1,67 \%$ & $\mathrm{Mn}$ & $857 \mathrm{ppm}$ \\
\hline $\mathrm{S}$ & $4510 \mathrm{ppm}$ & $\mathrm{Ti}$ & $10400 \mathrm{ppm}$ \\
\hline
\end{tabular}

de adición tal como se detalla en la Tabla 3. La muestra MII se preparó suponiendo que todo la ceniza dejada por el máximo reemplazo de combustible tradicional por alternativo, se incorpora al clinker. MI prevé la mitad de incorporación y MIII un exceso a fin de detectar tendencias.

Para la preparación de las diferentes muestras, se pesaron cuantitativamente las cenizas y el material crudo y se mezclaron en seco. Las muestras fueron pelletizadas y luego clinkerizadas en horno - mufla, con velocidad de calentamiento de $10{ }^{\circ} \mathrm{C} / \mathrm{min}$ hasta temperatura final de $1450{ }^{\circ} \mathrm{C}$ y mantenidas a esa temperatura durante una hora. La velocidad de enfriamiento también fue controlada a fin de asegurar la permanencia de las fases hidráulicas deseadas.

\subsection{Técnicas de análisis}

Los distintos clinkers sintetizados fueron molidos en un molino oscilante Herzog HSM 100 con cámara de acero al vanadio. Se molieron iguales cantidades de cada clinker durante igual tiempo a fin de hacer comparativo el ensayo. El tiempo de molienda se estableció de forma tal que la superficie específica quede dentro del rango utilizado en los cementos normales.

Sobre los clinkers molidos se realizaron los siguientes ensayos: Medición de la superficie específica por el método Blaine (IRAM 1623), medición de la temperatura de cono pirométrico equivalente, (TCPE) según IRAM 12507, análisis térmico diferencial (ATD/TG) y difracción de rayos-X 
Tabla 3. Porcentaje de adición y denominación de las distintas muestras.

\begin{tabular}{ccc}
\hline $0,03 \%$ & $0,06 \%$ & $0,24 \%$ \\
\hline MI & MII & MIII \\
\hline
\end{tabular}

(DRX). Para estos ensayos se utilizó un equipo NETZCH STA 409 y un difractómetro PHILIPS PW 3710, respectivamente.

A las diferentes edades de hidratación las pasta con $\mathrm{W} / \mathrm{C}=0,4$ fueron analizadas por ATD/TG y DRX. Se realizaron además, estudios de porosimetría por intrusión de mercurio y se midió la resistencia a la compresión sobre pastas a distintas edades (3, 7 y 28 días) utilizando una máquina J.J. INSTRUMETS modificada. Se realizaron además ensayos de calorimetría diferencial durante las primeras 48 h de hidratación.

\section{Análisis y discusión de resultados de los clinkers}

\subsection{Análisis por DRX}

El difractograma obtenido para el clinker de referencia se presenta en la Fig. 1, comparativamente con el correspondiente a MIII. En los mismos se pueden identificar todas las fases típicas del clinker portland como $\mathrm{C}_{3} \mathrm{~S}^{*}, \mathrm{C}_{2} \mathrm{~S}$ y $\mathrm{C}_{3} \mathrm{~A}$ entre otras.

Los difractogramas obtenidos de las clinkers sintetizados en presencia de cenizas de la mezcla combustible, en las proporciones especificadas, no mostraron la formación de nuevas fases o componentes que puedan detectarse por esta técnica.

Los principales componente del clinker muestran una mayor cristalinidad de sus fases cuando la incorporación es máxima $(0,24 \%)$ superando a la referencia. Los restantes clinkers con 0,03 y $0,06 \%$ de cenizas presentan cristalinidad semejante a la referencia, sugiriendo que el aporte de los elementos mineralizadores incorporados a través de las cenizas, no sigue una tendencia que pueda correlacionarse con la cantidad adicionada.

\subsection{Superficie específica}

En los materiales en polvo, el conocimiento de área específica es de suma importancia, ya que de ella dependerá su actividad fisicoquímica y además es una medida indirecta de su tamaño de partícula y por ende de su energía superficial. Para la medición de la superficie específica se utilizó un permeabilímetro Blaine por ser apropiado para este tipo de materiales y muy usado en la industria del cemento (IRAM 1623).

El comportamiento del clinker durante la molienda está directamente relacionado a la textura y estructura de los cristales formados durante la clinkerización ${ }^{17}$. Los resultados obte-
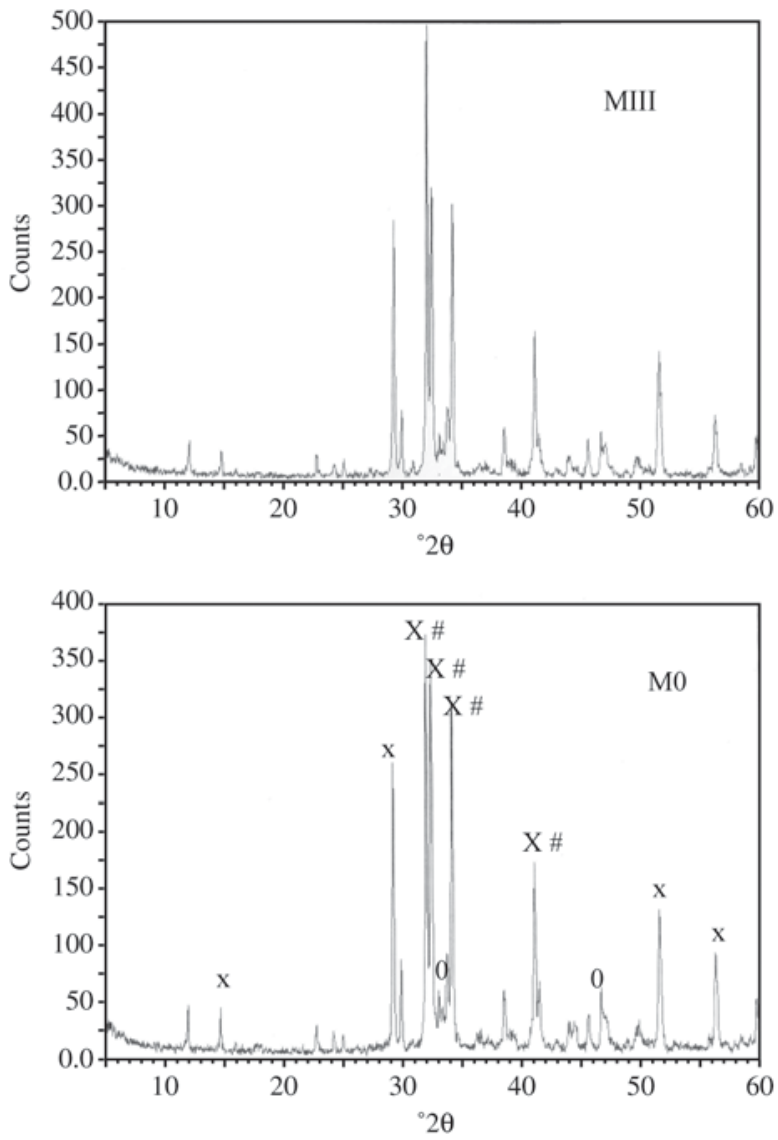

Figura 1. DRX clinkers Referencia (M0) y MIII: $\mathrm{X}=\mathrm{C}_{3} \mathrm{~S}, \#=\mathrm{C}_{2} \mathrm{~S}, 0=\mathrm{C}_{3} \mathrm{~A}$.

nidos para las distintas muestras se presentan en la Tabla 4.

Es interesante observar lo ocurrido en las muestras con cenizas del combustible alternativo. $\mathrm{Al}$ aumentar el porcentaje de incorporación de cenizas disminuye la superficie específica, a pesar de entregar igual energía por unidad de masa y tiempo en el proceso de molienda, indicando esto una mayor dificultad de molienda (material más duro). Podría pensarse que la adición de impurezas al material crudo afecta las propiedades fisicoquímicas del líquido formado durante la clinkerización, determinando la formación preferencial de algunas fases respecto de otras, afectando la microestructura del clinker ${ }^{6,18,19}$ como ya se vio en los DRX. Las mayor cantidad de fases cristalinas formadas (especialmente $\mathrm{C}_{3} \mathrm{~S}$ ) han generado la mayor dificultad de molienda que hemos observado.

\subsection{Temperatura de Cono Pirométrico Equivalente (TCPE)}

La sola medición de la TCPE no aporta información sobre la naturaleza de la clinkerización o las características

* Se usará la momenclatura de la Química del Cemento: C: $\mathrm{CaO}$, 
del producto final. En consecuencia, la información aportada por este ensayo se discutirá en forma comparativa entre la referencia y las diferentes muestras con adición de cenizas.

El ensayo se realizo sobre las mezclas crudas del material de referencia y con la adición de cenizas de mezcla combustible en los porcentajes de ensayo. Los resultados obtenidos se muestran en la Tabla 5.

Se observa en la tabla que dicha incorporación aumenta la TCPE en función del contenido de impurezas y los valores obtenidos son mayores que el de la referencia. Esto estaría indicando que su presencia hace al material más refractario y que este efecto es función de la cantidad de impurezas incorporadas

Además es interesante destacar en este ensayo que los conos no se doblaron a la temperatura señalada, como ocurre normalmente, sino que los mismos se fundieron en forma violenta, perdiendo totalmente su forma. Esto indica la formación de un fluido de menor viscosidad que en el caso de M0 tal como se visualiza en la Fig. 2.

Es importante observar también que en MI (mínima incorporación de cenizas) el cono funde mostrando que casi no existió deformación previa a la fusión - proceso de fusión muy rápido -, sin embargo a medida que aumentaba el

Tabla 4. Superficie Específica Blaine en $\mathrm{m}^{2} / \mathrm{kg}$.

\begin{tabular}{cc}
\hline Muestra & $\mathrm{m}^{2} / \mathrm{kg}$ \\
\hline M0 & 363 \\
MI & 370 \\
MII & 347 \\
MIII & 316 \\
\hline
\end{tabular}

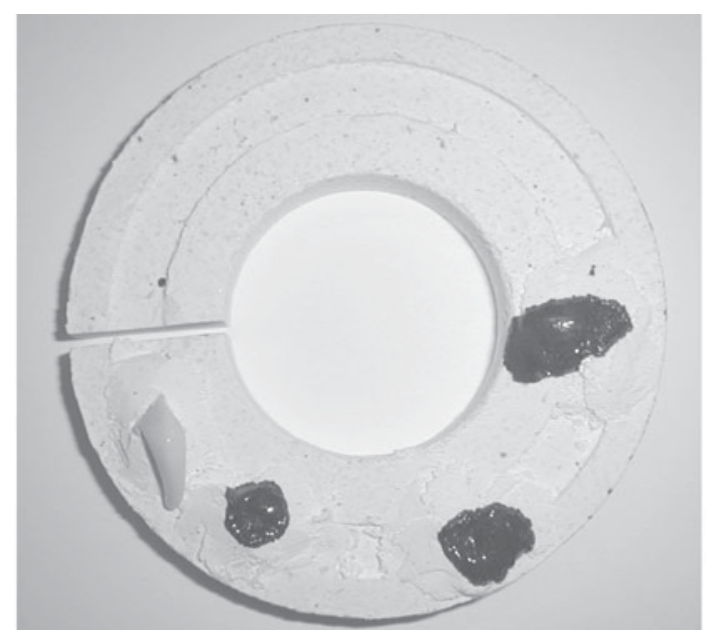

porcentaje de cenizas se detecta una mínima deformación previa a la fusión indicando que el efecto es función del porcentaje de cenizas incorporado.

Esta fusión violenta casi sin deformación podría traer complicaciones en los hornos de producción perfilándose como muy peligroso, pues puede ocasionar problemas en los refractarios debido a la penetración en los mismos de las fases fundidas (pegaduras). Sin embargo los procesos químicos en este medio fundido de baja viscosidad (aún en MIII), producen cristales más grandes por favorecer sus procesos de crecimiento. En el caso de MIII ya mencionado esto se tradujo en la formación de fases mejor cristalizadas y se vio reflejado en una mayor resistencia a la molienda.

\subsection{Análisis térmico}

Se realizó el análisis térmico diferencial y termogravimétrico en forma reversible (calentamiento- enfriamiento) y en iguales condiciones térmicas en que se elaboraron los clinkers sobre las distintas muestras de polvo crudo, con y sin las incorporaciones de cenizas de mezcla combustible en los porcentajes indicados anteriormente. El programa de temperatura utilizado fue el siguiente:

$20{ }^{\circ} \mathrm{C}$ a $1450{ }^{\circ} \mathrm{C}$ velocidad $10{ }^{\circ} \mathrm{C} / \mathrm{min}$.

$1450^{\circ} \mathrm{C}$ durante 1 hora

$1450{ }^{\circ} \mathrm{C}$ a $1350{ }^{\circ} \mathrm{C}$ velocidad $30{ }^{\circ} \mathrm{C} / \mathrm{min}$.

$1350{ }^{\circ} \mathrm{C}$ a temperatura ambiente $10{ }^{\circ} \mathrm{C} / \mathrm{min}$.

En el diagrama de ATD-TG para la referencia (Fig. 3), durante el calentamiento y luego de la estabilización del sistema aparece un primer pico endotérmico de gran magnitud correspondiente a la descomposición de la caliza. Dicho pico se ubica a la temperatura de $922,3{ }^{\circ} \mathrm{C}$ en la muestra de referencia. Superada la decarbonatación y siempre durante el calentamiento, aparece la zona de reacción del $\mathrm{CaO}$ con los silicatos y silico-aluminatos, se presenta como

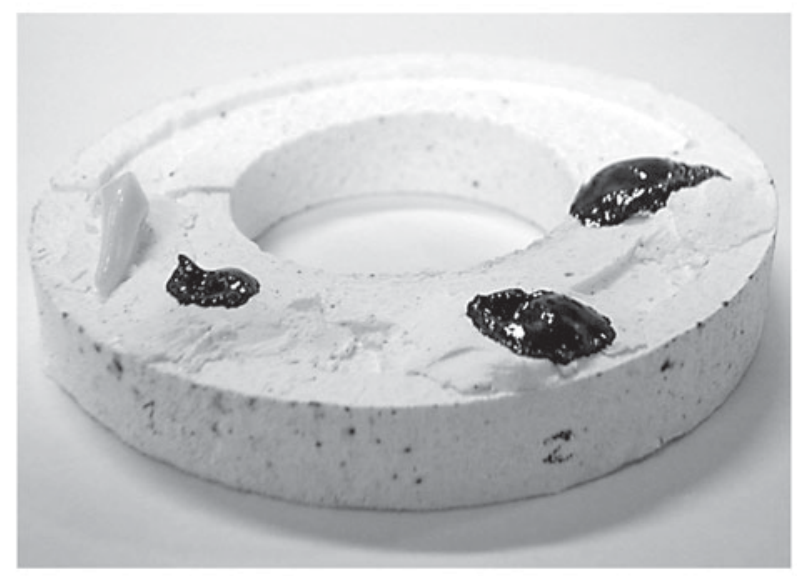

Figura 2. Conos de la serie M (de izquierda a derecha: Cono pirométrico de referencia - Orton-, MI, MII y MIII). 
Tabla 5. Temperatura equivalente en función de las impurezas adicionadas.

\begin{tabular}{lc}
\hline Muestra & $\begin{array}{c}\text { Temperatura } \\
\text { equivalente }\left({ }^{\circ} \mathrm{C}\right)\end{array}$ \\
\hline M0 & 1520 \\
MI & 1530 \\
MII & 1540 \\
MIII & 1550 \\
\hline
\end{tabular}

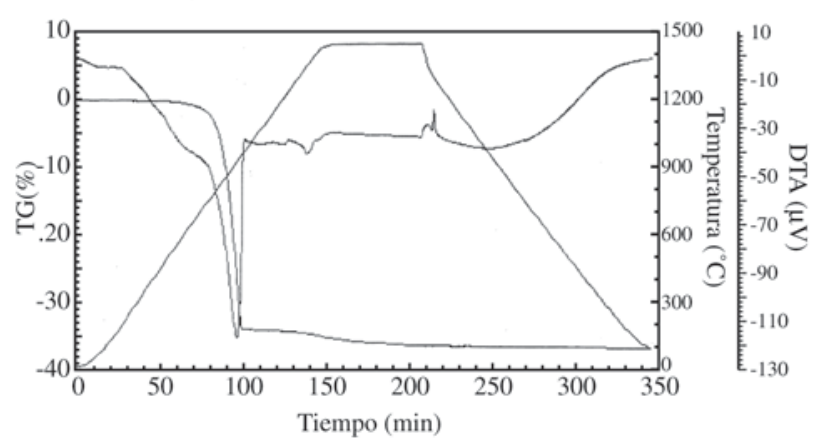

Figura 3. DTA-TG correspondiente a M0.

una banda endotérmica en la zona de 950 - $1000{ }^{\circ} \mathrm{C}$. En las muestras analizadas no está bien definida como pico, ya que estas reacciones en estado sólido son mayoritariamente de control difusional, la que genera bandas chatas y anchas en lugar de picos.

Al seguir aumentando la temperatura se observa un pico endotérmico en $1345,6^{\circ} \mathrm{C}$ para $\mathrm{M} 0$, asignable a la formación de fase fundida (clinkerización).

Durante el enfriamiento se puede ver en la curva de ATD un único pico agudo exotérmico a $1285,1^{\circ} \mathrm{C}$ en la referencia, asignable a la solidificación de la fase líquida formada durante la clinkerización ${ }^{20}$.

Los estudios por ATD/TG de las muestras con incorporación de las diferentes adiciones presentan igual aspecto general, con ligeros corrimientos en las temperaturas de clinkerización y cristalización, tal como se muestra en la Tabla 6.

La temperatura de clinkerización para las muestras de la serie $\mathrm{M}$, presentan una tendencia similar a la de los ensayos de TCPE es decir: $\mathrm{MIII}>\mathrm{MII}>\mathrm{M} 0$, con un $\Delta \mathrm{T} \approx 5^{\circ} \mathrm{C}$ entre cada uno. Las temperaturas de solidificación son similares entre MII y M0 pero es mayor para MIII, coincidiendo con la secuencia dada para la clinkerización.

El ligero aumento de la temperatura de clinkerización y la variación de la temperatura de solidificación observada en algunos casos indica un corrimiento de la zona de formación y permanencia de la fase fundida. Esto justifica las
Tabla 6. Temperaturas de reacción para las diferentes muestras.

\begin{tabular}{lcc}
\hline Muestra & $\begin{array}{c}\text { Temperatura de } \\
\text { clinkerización }\left({ }^{\circ} \mathrm{C}\right) \\
\text { Calentamiento }\end{array}$ & $\begin{array}{c}\text { Temperatura de } \\
\text { solidificación }\left({ }^{\circ} \mathrm{C}\right) \\
\text { Enfriamiento }\end{array}$ \\
\hline Referencia & 1345,6 & 1285,1 \\
MII & 1350,6 & 1284,5 \\
MIII & 1354,9 & 1290,0 \\
\hline
\end{tabular}

modificaciones estructurales que fueron detectadas por DRX, diferentes temperaturas de CPE y superficie específica de material molido con respecto a la referencia.

\section{de los clinkers hidratados}

\subsection{Análisis por DRX}

El seguimiento de la hidratación a edades tempranas (1 a 28 días) por DRX no mostró diferencias significativas en lo que respecta a la naturaleza de la hidratación de las muestras aditivadas con relación a la referencia, es decir generan los mismos productos de hidratación en tiempos similares.

En general el avance de la hidratación, seguida a través de la disminución de los picos de $\mathrm{C}_{3} \mathrm{~S}$ y $\mathrm{C}_{2} \mathrm{~S}$ y el crecimiento de los correspondientes al $\mathrm{CH}$, es menor en las muestras aditivadas que en M0, a edades tempranas. Esto puede vincularse a fenómenos superficiales dada la mayor superficie específica de M0 respecto a las restantes muestras.

Sin embargo a 28 días los picos de CH de MIII superan a la referencia y a las demás muestras de la serie, indicando claramente un mayor avance de la hidratación. Por esto cabría esperar, mayores valores de resistencia a la compresión a estas edades.

\subsection{Calorimetría diferencial}

La cinética de los procesos de hidratación podría relacionarse en estos casos con dos factores determinantes: la superficie específica y la presencia de impurezas. A mayor superficie específica menores tiempos de hidratación, en especial en aquellas etapas iniciales de mojado y disolución, vinculadas estrechamente a la superficie expuesta. En cuanto a la presencia de impurezas, por ejemplo, alita y belita se hidratan más rápidamente que las correspondientes fases puras $^{21}$. Estos factores actúan conjuntamente en estos casos y no siempre en el mismo sentido.

En la Fig. 4a se presentan las curvas calorimétricas correspondientes a la serie $\mathrm{M}$, donde se puede observar comparativamente la modificación de los tiempos de las etapas de solubilización e inicio de la precipitación de los aluminatos, primer y segundo pico respectivamente.

En primera instancia estas diferencias, que tienen origen en el retraso de la etapa inicial de hidratación, pueden 
atribuirse a una menor actividad superficial a medida que disminuye la superficie específica. Estos tiempos disminuyen al pasar de MIII a MII y MI. Recuérdese que al pasar de MI a MIII la superficie específica Blaine varió de 370 a $316 \mathrm{~m}^{2} / \mathrm{kg}$ a pesar de entregarles igual cantidad de energía. Se suma a este efecto el mayor contenido de impurezas que aumenta al pasar de MI a MIII actuando ambos efectos en el mismo sentido.

Al comparar estas curvas calorimétricas con la correspondiente a la referencia, el atraso de los tiempos de solvatación e inicio de la hidratación de los aluminatos debe vincularse más con la presencia de impurezas incorporadas por las cenizas que a los valores de superficie específica, a pesar que esta etapas son de control químico y están fuertemente vinculadas a la superficie de reacción expuesta. Estas impurezas marcan las diferencias entre M0 y MI tal como se observa en la Fig. 4b, donde este aspecto es determinante ya que ambos poseen una superficie específica similar.

Previo al tercer máximo, se observa un período de ace-

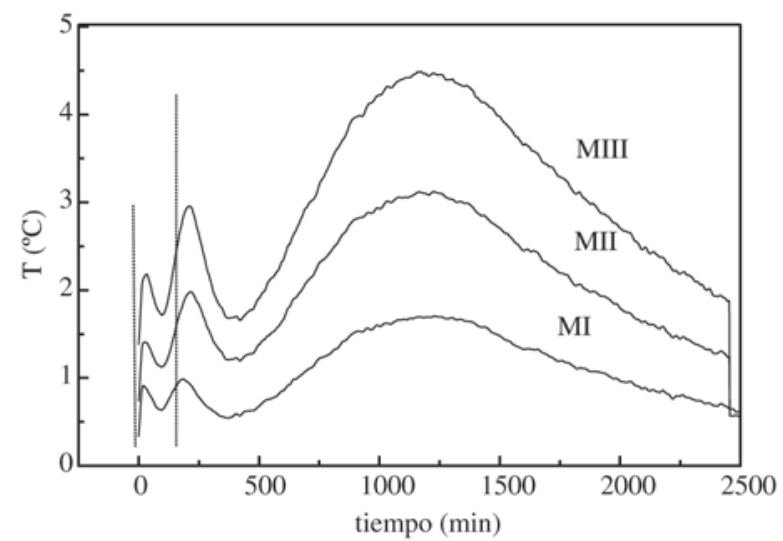

(a)

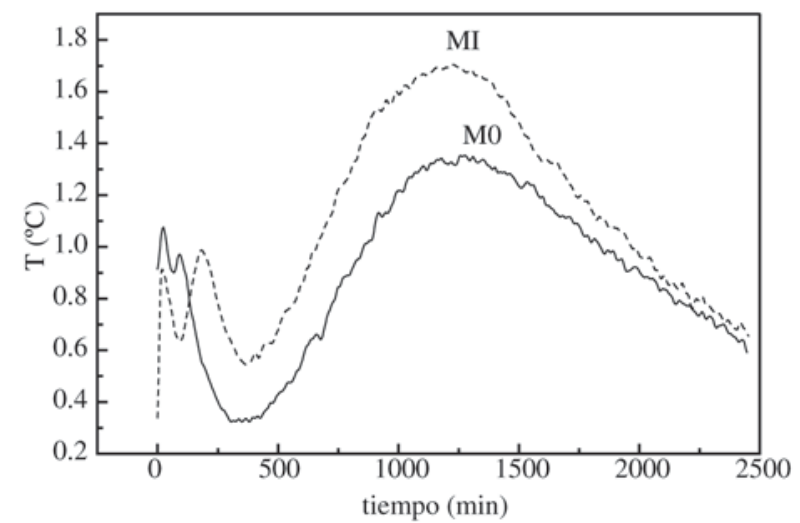

(b)

Figura 4. Curvas calorimétricas. (a) Serie MI, MII, MIII (b) M0, MI. leración que determina en su punto máximo el final de los procesos de formación de núcleos de los hidratos y el inicio de la ganancia de resistencia de la pasta debido al crecimiento y entrecruzamiento de estos últimos. Por ello este máximo se suele correlacionar con el final del fraguado ${ }^{22}$. Este proceso controlado químicamente se origina por la formación de productos de hidratación a partir de los silicatos.

Aparentemente $^{21}$, el $\mathrm{CH}$ cristaliza desde la solución, mientras que el C-S-H se desarrolla sobre la superficie de los granos de $\mathrm{C}_{3} \mathrm{~S}$, cubriéndolos. Así al continuar la hidratación crece la capa formada e interfiere, actuando como barrera, entre el material sin hidratar y el agua. Por ello el agua debe difundir para alcanzar el material anhidro y los iones difundir en el sentido contrario para alcanzar las zonas de crecimiento, esto determina un período de desaceleración controlado química y difusionalmente. El movimiento de los iones a través de las capas de C-S-H determina la velocidad de reacción de esta etapa, la cual viene controlada por difusión. Estos procesos son más lentos a medida que crece el ancho de la barrera, en consecuencia se completan a muy largo plazo. Esta etapa determina la velocidad de ganancia de resistencia

La mayor formación de geles en la etapa inicial de hidratación de M0 disminuye la velocidad de ganancia de resistencia (etapa de control difusional) con respecto a las muestras de la serie aditivadas. Estas últimas poseen una pendiente mayor lo que haría pensar en una mayor velocidad de ganancia de resistencia a edades tempranas (primeras 48 horas de hidratación) que la referencia y al pasar de MI a MIII. Estas diferencias de todas formas, no tendrán influencia en las propiedades mecánicas del material medidas a mayor tiempo de hidratación.

\subsection{Análisis térmico diferencial}

Se realizó un análisis de las muestras hidratadas durante 45 días $($ con w/c $=0.4$ ) por ATD/TG. Las mismas fueron calentadas entre 25 y $700{ }^{\circ} \mathrm{C}$.

Observando los resultados se detectan dos picos endotérmicos bien definidos los mismos son debidos a la deshidratación del gel C-S-H y a la descomposición del CH. El primero de ellos se observa en la zona de $130-200{ }^{\circ} \mathrm{C}^{23-25}$ como una ancha banda que se corre a mayores temperaturas con el tiempo de hidratación ${ }^{26}$. La forma redondeada de este pico es típica de sustancias que pierden agua sin que se produzca un sensible cambio estructural ${ }^{27}$. Este cambio esta acompañado por una gran pérdida de masa.

El segundo pico endotérmico observado a $530{ }^{\circ} \mathrm{C}$ aproximadamente se atribuye a la descomposición del $\mathrm{CH}$ cristalino producido por la hidratación de las fases silicato ${ }^{26,28}$. La pérdida de masa que acompaña a esta descomposición puede relacionarse con el avance de la hidratación. Los cambios descriptos se muestran en la Fig. 5 para M0. 
La Tabla 7 muestra los resultados obtenidos para las distintas muestras analizadas.

En las muestras aditivadas con la mezcla combustible, las temperaturas de picos correspondiente a los geles son similares a M0, salvo en MIII en la cual es ligeramente superior $\left(1,5^{\circ} \mathrm{C}\right)$. En el segundo pico endotérmico las temperaturas son menores que para M0 pero incrementándose de MI a MIII. Las variaciones de masa muestran algo menos de geles en MIII que en la muestra original y un tenor similar de CH (2do. Pico de TG) que en los otros casos lo cual coincide con lo ya visto por calorimetría diferencial. De todas formas, los resultados obtenidos por esta técnica -diferencias entre ellos - no son tan significativas como para esperar una gran influencia en las propiedades mecánicas.

\subsection{Porosimetría}

Los ensayos de porosimetría se realizaron sobres muestras hidratadas $(\mathrm{w} / \mathrm{c}=0,4)$ durante 45 días. Varios autores ${ }^{29,30}$ coinciden en que la distribución de tamaños de poros es el mejor criterio para evaluar las características de los huecos capilares mayores de $50 \mathrm{~nm}$ de una pasta de cemento hidratada. Esta porosidad va en detrimento de la resistencia e impermeabilidad, mientras que los huecos menores de $50 \mathrm{~nm}$ tienen mayor influencia sobre la contracción por secado y el creep.

Las muestras ensayadas prácticamente no presentaron macroporosidad entre 30 y $5 \mu \mathrm{m}$. La macroporosidad influye directamente sobre la resistencia mecánica del material.
En la Fig. 6 se presentan los resultados de porosidad de las diferentes muestras ensayadas en el rango de $5-5000 \mathrm{~nm}$ (50 a $50000 \AA$ ) y se señala la línea de $50 \mathrm{~nm}$. Las diferencias encontradas en la zona mayor a $50 \mathrm{~nm}$ no son significativas y en consecuencia no influirán en la resistencia mecánica del material. En la zona menor a $50 \mathrm{~nm}(500 \AA$ A ) las muestras de la serie M presentan un mayor volumen total de poros que la referencia y dentro de ellas la curva correspondiente a MIII se encuentra por encima, indicando que la muestra con mayor cantidad de impurezas es quien presenta mayor microporosidad.

Las consecuencias de era distribución de tamaño de poros no se vio relacionada con las propiedades mecánicas medidas.

\subsection{Resistencia mecánica}

Los resultados de resistencia a la compresión, obtenidos como promedio de cinco ensayos, a la edad de 3, 7, 14 y 28 días se muestran en la Fig. 7.

A temprana edad, 3 días, las muestras se comportaron en forma diferente a lo esperado, acusando una mayor hidratación la muestra MII que las restantes, inclusive M0 a pesar que su mayor valor de superficie específica hacía pensar lo contrario. Sin embargo es necesario aclarar que las diferencias entre los valores máximos y mínimos respecto a sus valores medios fue a esa edad de $25 \%$, lo que podría

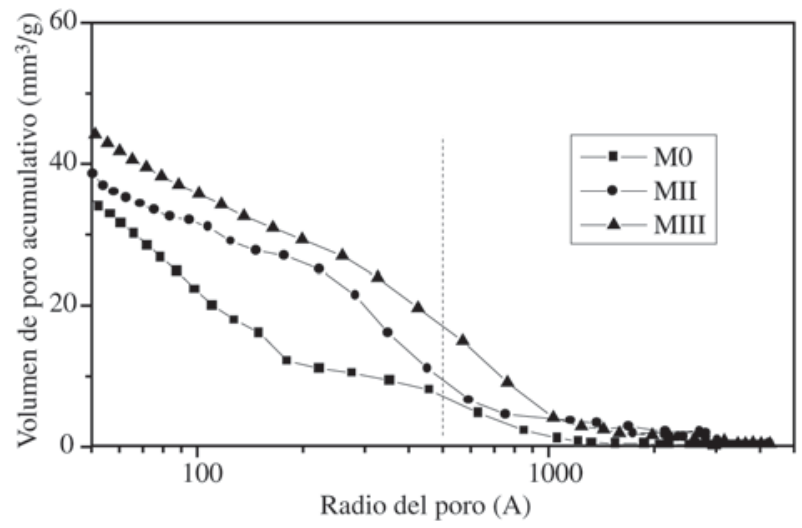

Figura 6. Distribución de tamaño de poro, Meso-Microporos.

Figura 5. ATD-TG correspondiente a M0 hidratada por 45 días.

Tabla 7. Resultados ATD/TG de los diferentes clinkers hidratados.

\begin{tabular}{lccccccc}
\hline Muestra & $\begin{array}{c}\Delta \mathrm{m}(\%) \\
(\text { total })\end{array}$ & $\begin{array}{c}1^{\circ} \text { Pico } \\
\text { ATD }(\mathrm{C}-\mathrm{S}-\mathrm{H})\end{array}$ & \multicolumn{2}{c}{$2^{\mathrm{o}}$ Pico } & \multicolumn{2}{c}{$1^{\text {er }}$ pico TG } & \multicolumn{2}{c}{$2^{\text {do }}$ Pico TG } \\
& & $\left({ }^{\circ} \mathrm{C}\right)$ & $\left({ }^{\circ} \mathrm{C}\right)$ & $\Delta \mathrm{m}(\%)$ & $\left({ }^{\circ} \mathrm{C}\right)$ & $\Delta \mathrm{m}(\%)$ & $\left({ }^{\circ} \mathrm{C}\right)$ \\
\hline M0 & 26,3 & 147,0 & 540,4 & 18,6 & 133,7 & 6,9 & 534,1 \\
MII & 26,3 & 147,3 & 534,0 & 18,2 & 130,8 & 6,3 & 527,7 \\
MIII & 26,2 & 148,5 & 538,9 & 18,4 & 134,2 & 6,8 & 532,5 \\
\hline
\end{tabular}




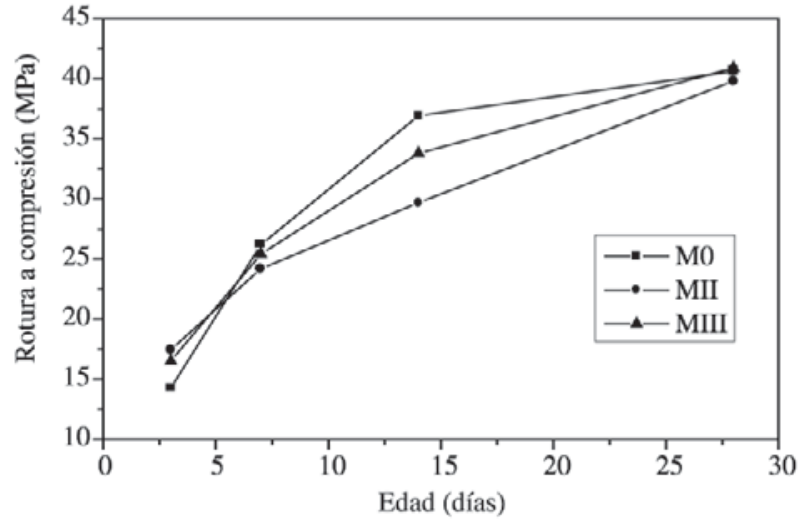

Figura 7. Rotura a la compresión, de pasta in MPa.

enmascarar diferencias observadas.

A partir de 7 días, disminuyó considerablemente la dispersión (8\%) y los valores de resistencia a la compresión de la serie M se ubicaron siempre por debajo de MIII y este por debajo de M0. A los 28 días de hidratación todas las muestras acusaron un comportamiento mecánico muy homogéneo.

Esto pone de manifiesto que las diferencias medidas por otras técnicas en cuanto a la velocidad de ganancia de resistencia temprana de las muestras aditivadas con respecto a la referencia no se traducen en un significativa diferencia de la resistencia a la compresión. Esto favorece notablemente al uso de esta tecnología.

\section{Conclusiones}

La incorporación de cenizas provenientes de los combustibles alternativos al proceso de clinkerización de cemento Portland, introduce al sistema óxidos y sales metálicas en porcentajes variables y algunos en el orden de trazas.

Su presencia, y en función del porcentaje incorporado, modifica las propiedades del fundido generado durante la clinkerización, lo cual beneficia los procesos de difusión obteniéndose fases mejor cristalizadas. Por otro lado esto hace que se requiera un buen control de temperatura en la zona de clinkerización del horno, pues un exceso podría fluidificar demasiado el clinker, ocasionando serios inconvenientes en el revestimiento del horno. Se suma a esto que la mayor cristalinidad de las fases obtenidas en el clinker, trae aparejado mayor requerimiento energético de molienda.

Durante la hidratación del clinker sintetizado en presencia de cenizas, se detectaron variaciones en la velocidad de hidratación, pero no en la naturaleza de los productos obtenidos respecto a la referencia. Esto determinó algunas ligeras modificaciones en la distribución y tamaño de po- ros, cantidad de geles formados etc. Pero en ningún caso esto parece tener significativa influencia en las propiedades mecánicas del cemento obtenido, a la edad de 28 días.

La incorporación de estos combustibles residuales, en los porcentajes y condiciones de este ensayo, no afectan negativamente las propiedades del clinker.

\section{Referencias}

1. Trovaag K., Hazardous incineration in a cement kiln. World Cement, v. 14, n. 2, p. 59-62, 1983.

2. Mollah, M.Y.A.; Vepati, R.K.; Lin, T-C.; Cocke, D.L. The interfacial chemistry of solidification/ stabilization of metals in cement and pozzolanic material systems, Waste Management, v. 15, n. 2, p. 137-148, 1995.

3. Díez,k J.M.; Madrid, J.; Macías, A. Characterization of cement-stabilized Cd wastes, Cem. Concr. Res., v. 27, n. 4, p. 479-485, 1997.

4. Asavapisit, S.; Fowler, G.; Cheeseman, C.R. Solution chemistry during cement hydration in the presence of metal hydroxide wastes, Cem. Concr. Res., v. 27, n. 8, p. 1249-1260, 1997.

5. Madrid, J.; Diez, J.M.; Goñi, S.; Macías, A. Durability of cement matrices used for stabilization of hazardous wastes, International Conference Durability of Concrete, Ed. V.M. Malhotra, p. 1527-1551, 1997.

6. Kakali, G.; Kasselouri, V.; Parissakis, G. Investigation of the effect of $\mathrm{Mo}, \mathrm{Nb}, \mathrm{W}$ and $\mathrm{Zr}$ oxides on the formation of portland cement clinker, Cem. Concr. Res., v. 20, n. 1, p. 131-138, 1990.

7. Mollah, M.Y.A.; Hess, T.; Tsai Y-N., An FTIR and XPS investigation of the effects of carbonation on the solidification/stabilization of cement based systemsportland type V with zinc, Cem. Concr. Res., v. 23, n. 4, p. 773-784, 1993.

8. Tashiro, C.; Takahashi, H.; Kanaya, M.; Hirakida, I.; Yoshida, R. Hardening property of cement mortar adding heavy metal compound and solubility of heavy metal from hardened mortar, Cem. Concr. Res., v. 7, n. 3, p. 383-290, 1977.

9. Odler, I.; Schmidt, O. Structure and properties of portland cement clinker doped with zinc oxide, J. Am. Ceram. Soc., v. 63, n. 1-2, p. $13-16,1980$.

10. Hanna, R.A.; Barrie, P.J.; Cheeseman, C.R.; Hills, C.D.; Buchler, P.M.; Perry, R. Solid state ${ }^{29} \mathrm{Si}$ and ${ }^{27} \mathrm{Al}$ and FTIR study of cement pastes containing industrial wastes and organics, Cem. Concr. Res., v. 25, n. 7, p. 14351444, 1995.

11. Murat, M.; Sorrentino, F. Effect of large additions of $\mathrm{Cd}, \mathrm{Pb}, \mathrm{Cr}, \mathrm{Zn}$ to cement raw meal on the composition and the properties of the clinker and the cement, Cem. Concr. Res., v. 26, n. 3, p. 377-385, 1997.

12. Mollah, M.Y.A.; Parga, J.R.; Cocke, D.L. An infrared 
spectrocopic examination of cement-based solidificaion/ stabilization systems-portlad types V and IP with zinc, J. Environ. Sci. Health, v. 27, n. 6, p. 1503-1519, 1992.

13. Mollah, M.Y.A.; Tsai, Y-N.; Cocke, D.L. An FTIR investigation of cement based solidification/stabilization systems doped with cadmium, J. Environ. Sci. Health, v. 27, n. 5, p. 1213-1227, 1992.

14. Kakali, G.; Tsivilis, S.; Tsialtas, A. Hydration of ordinary portland cements made from raw mix containing transition element oxides, Cem. Concr. Res., v. 28, n. 3, p. 335- 340, 1998.

15. Bolio-Arceo, H.; Glasser, F.P.; Zinc oxide in cement clinkering: part 1. Systems $\mathrm{CaO}-\mathrm{ZnO}-\mathrm{Al}_{2} \mathrm{O}_{3}$ and $\mathrm{CaO}-$ $\mathrm{ZnO}-\mathrm{Fe}_{2} \mathrm{O}_{3}$, Advances in Cement Research, v. 10, n. 1, p. 25-32, 1998.

16. Maringolo, V.; Suto, A.J.; Barbosa A.M., Kihara, Y.; Vazarcacour, N.N.; Comparative study of clinker burned with up to $30 \%$ substitution of fuel for waste fuel. Proceedings, $5^{\circ}$ Congresso Brasileiro de Cimento, May 03, p. 144, 1999.

17. Tsivilis, S.; Kakali, G. A study on the grindability of portland cement clinker containing transition metal oxides. Cem. Concr. Res., v. 27, n. 5, p. 673-678, 1997.

18. Trezza, M.A.; Scian, A.N.; Burning waste as an industrial resource. Their effect on portland cement clinker. Cem. Concr. Res., v. 30, n. 1, p. 137-144, 2000.

19. Kakali, G.; Parissakis, G. Investigation of the effect of $\mathrm{Zn}$ oxide on the formation of portland cement clinker. Cem. Concr. Res., v. 25, n. 1, p. 79-85, 1995.

20. Blanco Varela, M.T.; Palomo, A.; Vazquez, T. Effect of fluorspar on the formation of clinker phases. Cem. Concr. Res., v. 14, n. 3, p. 397- 406, 1984.

21. Mindess, S.; Young, J.F. Concrete. Prentice Hall Inc., New York, USA . 1981.

22. Alumno Rosetti, V.; Medici, I. Inertization of toxic metals in cement matrices: Effects on hydration, setting and hardening. Cem. Concr. Res., v. 25, n. 6, p. 1147-1152, 1995.

23. El-Dinamony, H.h Sharara, A.M.; Helmy, I.M.; Abd ElAleem, S. Hydration characteristics of $\beta-\mathrm{C}_{2} \mathrm{~S}$ in the presence of some accelerators. Cem. Concr. Res., v. 26, n. 8, p. 1179-1187, 1996.

24. Mackenzie, R.C. - Editores, Diferential Thermal Analysis - v. 2. Academic Press, USA, 1972.

25. Klimesch, D.; Ray, A.; Sloane, B. Autoclaved cementquartz pastes: the effects on chemical physical properties when using ground quartz with different surface areas. Cem. Concr. Res., v. 26, n. 9,p. 1399-1408, 1996.

26. Oriol, M.; Pera, J.; Pozzolanic activity of metakaolin under microwave treatment. Cem. Concr. Res., v. 25, n. 2, p. 265-270, 1995.

27. Taylor, H.F.W. La química de los cementos, ediciones Urmo, España, 1967.

28. Bensted, J. Some applicationes of IR and Raman spectroscopic in cement chemistry - part II. Cement Technology, v. 5, n. 4, p. 378-382, 1974.

29. Metha, K.P.; Monteiro, P.J.M. Concrete, structure, properties and materials. Prentice Hall, New York, 1993.

30. Neville, A.M. Tecnología del concreto. Pentice Hall, New York, 1981. 